\title{
Emotional Intelligence in the Educational and Therapeutic Community in Nepal
}

\author{
Christopher Rybak* \\ Chhori Laxmi Maharjan $^{* *} \&$ Anubha Adhikari ${ }^{* *}$
}

Abstract

Emotional intelligence has been gaining increased attention in both the educational as well as therapeutic communities of Nepal. Research suggests that development of emotional intelligence has a significant influence on an individual's achievements including academic success and is seen as a key aspect of positive development (Mayer, Salovey, \& Caruso, 2004).

Ankur Counseling Center in Kathmandu was established in 2006, primarily to work with children who have experienced great stress in their lives. Counselors at Ankur have been using play and sand tray therapy to understand the emotional lives of their clients, as well as to assist the process of emotional healing.

\section{Emotional intelligence in the educational and therapeutic community of Nepal}

The recognition of the importance of emotional intelligence and its development through maturation and education has been growing in recent years. This article will offer a definition of emotional intelligence and describe some of the basic research and application of emotional intelligence principles to education. This article will also describe some therapeutic interventions designed to promote emotional healing and further the development of emotional intelligence by children who have suffered through emotionally disruptive experiences.

\section{What is emotional intelligence?}

Emotional intelligence includes the ability to be self-aware of one's emotional state, to make meaning of emotional reactions, and to regulate and manage emotional reactions (Mayer \& Salovey, 1990). Exploration of the meaning and measurement of emotional intelligence has been of intensifying interest in the educational community.

Mayer, Salovey, and Caruso (2004) identified four primary components to emotional intelligence: 1) perception of emotions, 2) facilitating thought processes through the use of emotion, 3) emotional understanding, and 4) the ability to effectively manage emotions. Emotional intelligence is positively correlated with positive social relationships with others and negatively associated with aggression and violence toward others. Higher emotional intelligence suggests

* Dept. of ELH, Bradley University, Peoria, IL USA

** Ankur Counseling Center, Ekantikuna, Lalitpur, Nepal. 
36 Christopher Rybak, Chbori Laxmi Maharjan \& Anubha Adhikari

someone less likely to engage in personally self- destructive, violent, or addictive behaviors (Mayer, Salovey \& Caruso, 2004).

Goleman (1995) suggested five dimensions of emotional intelligence that include: 1) selfidentification of emotions; 2) Handling emotions in an appropriate manner; 3) Using emotions in an appropriate and productive manner; 4) ability to understand the emotions of others; and 5) Ability to develop effective relationships. Goleman's list specifically incorporated the aspect of relationship in addition to dimensions similar to those described by Mayer, Salovey and Caruso (2004).

Characteristics of higher levels of emotional intelligence include: 1) the ability to forego short-term pleasures for longer term well-being; 2) consideration of both the individual as well as others involved in the situation; 3) the type of emotions to experience are dependent on the situational context. Emotional regulation can occur at different levels of consciousness and the mechanism of regulation will match the consciousness level. Basic emotional orientation will beenacted atanon-consciouslevelofemotional regulation. At a low level of consciousness the mechanism will be characterized by both an open acceptance of the flow of emotion as well as an ability to view the situation from a perspective that disposes the emergence of both appropriate and effective emotions. At the highest level of consciousness, emotions are managed through keen self-observation and moral aplomb (Mayer \& Salovey, 1995).

\section{Emotional intelligence in education}

The skill of emotional self-regulation is considered an essential aspect of a child's ability to adapt successfully to the school environment (Posner \& Rothbart, 2007). Research has shown that children with average to high levels of emotional intelligences obtain higher grades and cause teachers fewer concerns about their behaviors (Qualter, Whitely, Hutchison, \& Pope, 2007).
In other research, children who scored higher when assessed for trait emotional intelligence were found to miss school less frequently and to be rated by teachers as having more advanced social skills when dealing with others (Mavroveli, Petrides, Sangoureau, \& Furnham, 2009). For children, "It has become increasingly clear that social and emotional skills are the prerequisite students require before they are in a position to access academic material presented in the classroom" (Romasz, Kantor, \& Elias, 2004, p. 92). If students fail to develop such skills, they will be less capable to meet the stresses and complex challenges of adulthood. As adolescents and adults they will have greater susceptibility to peer pressures to take drugs or participate in other dangerous and illicit activities. Additionally, they will have fewer options for resolving conflicts and more likely respond with violence (Romasz, Kantor, \& Elias, 2004).

\section{Emotional intelligence of educators and counselors}

As key aspects of such challenges, the level of emotional intelligence of educators and counselors merit particular attention since they are at the forefront of working with children, adolescents, and adults with the developmental process. The emotional intelligence of educators has a direct impact on the academic experiences and performances by students. In order to assess and support the development of emotional intelligence for their students, educators must have a clear recognition of emotional intelligence and how it is manifested in the classroom and other interpersonal situations (Kremenitzer, 2005).

Similarly for counselors who themselves regularly interact with students and children in emotionally charged situations, the level of emotional intelligence of counselors factors in to their therapeutic competence and thus plays a potentially significant role in the developmental process for children (Easton, Martin, \& Wilson, 2008). Indeed, emotional intelligence has been 
identified as one of the defining characteristics of counselors (Martin, Easton, Wilson, Takemoto, \& Sullivan, 2004). Researchers found a significant relationship between the level of counselor emotional intelligence and counselor ability to express empathy with clients. For counseling to be effective, counselors must be able to empathically identify the emotions experienced by their clients and also recognize their own emotional reactions to remain engaged but without themselves becoming emotionally overwhelmed by the experience (Miville, Carlozzi, Gushue, Schara, \& Ueda, 2006).

\section{Development of emotional intelligence}

Zeidner, Mathews, Roberts and MacCann (2003) identified three key aspects in the development of emotional imtelligence. Biological tendencies are relevant to emotional expression and management, learning rules about the expression of emotions is a key part of the socialization process, and the development of self-awareness promotes emotion identification as well as the management of emotional responses (Zeidner, Mathews, Roberts, \& MacCann, 2003).

\section{Biological basis}

Each person is born with certain basic biological tendencies relative to their emotional temperament. These tendencies have to do with the intensity with which various emotional responses are experienced and the degree to which such emotional responses may or may not be inhibited (Zeidner, Mathews, Roberts \& MacCann, 2003). Even with these basic tendencies of temperament, brain functions in general are imbued with the property of neuroplasticity. Neuroplasicity is the ability of the brain to change through experience. The connections between neurons are strengthened through repeated use and experience, thus skills are not only refined through practice, but the relevant neural connections are strengthened through familiarity (Siegel, 2007).

A relatively recent finding from research in neuroscience is the discovery of mirror neurons in the brain. Mirror neurons play a key role in the chain of inner events in that one person resonates to the perceived emotional state of another individual resulting in somatic reactions that are then felt and understood as being representative of the emotional state being experienced by another. Through this process, one's inner world becomes more or less attuned to the inner world perceived of others. These neurons appear to have a fundamental role for interpersonal relationships as these specialized neurons help one to resonate to the emotional state of others by representing the intentions of others and initiating a matching of these perceptions in one's own body. As such, mirror neurons are integral to the entire process of developing empathy for others and central to one's sense of social connectedness (Siegel, 2007). One learns to sense the intentions and goals of others through the neuropsychological mechanisms initiated through the activity of the mirror neurons. Although it is not yet clear whether mirror neurons are available at birth, it is clear that they develop and are modified throughout life with new experiences (Rizzolatti \& Fabbri-Destro, 2008).

Research using neuroimaging techniques suggests that by the age of adolescence those individuals who have developed a higher degree of emotional intelligence have a more efficient response within their brains to emotionally stimulating images as compared to individuals who scored lower on measures of emotional intelligence (Killgore $\&$ Yurgelun-Todd, 2007). The efficient brain response suggests that those who have developed a higher level of emotional intelligence may be more focused in an appropriate way toward responding to emotionally stimulating situations. Mirror neurons are prime examples of the neuroplasticity of the brain (Rizzolatti \& FabbriDestro, 2008).

\section{Socialization process}

As mirror neurons appear to be an integral part of the socialization process, research also suggests that 
38 Christopher Rybak, Chbori Laxmi Maharjan \& Anubha Adbikari

mirror neurons are modified through experiences. Such modifications appear to be initiated through observation of the intentional actions of others but are particularly strengthened when the similar actions are also performed (Rizzolatti \& Fabbri-Destro, 2008). This suggests a particular neuropsychological mechanism component of the modeling process that caregivers and others may have on a developing child.

Additionally, as an infant grows and begins to mature, the socialization process through contact with parents, family, and others begins to shape the way in which emotions are regulated (Zeidner, Mathews, Roberts, \& MacCann, 2003). The regulation mechanisms can include shutting down through inhibition or seeking expression and resolution of emotions through contact with caregivers. The growing child begins to learn the rules of culture and family. At this stage the child may not be especially reflective or purposeful in the selection of ways to manage emotions.

Preliminary research suggests that a moderate level of parental support for emotional expression by children is associated with greater emotional regulation by the children as they mature. Low levels of support may not offer a child sufficient opportunity to explore emotional reactions, while only high levels of support may not give a child experience with moderating intensive emotions short of impulsive expression. Variable levels of emotional support may also help a child learn that emotional intensity varies from person to person and situation to situation (McElwain, Halberstadt \& Volling, 2007).

\section{Self-reflective abilities}

With further development, children begin to gain in their capacity for self-awareness. They can reflect more on their emotional lives and make more purposeful choices regarding the means of expression, suppression, or inhibition of emotions. The growing person develops ways of finding solutions to issues that arise relative to their emotional experiences (Zeidner,
Mathews, Roberts, \& MacCann, 2003). Next research that was designed to examine the issue whether interventions such as training programs can actually improve self-reflective abilities and other aspects of emotional intelligence will be described.

\section{Training to enhance emotional intelligence}

Working with three and four year old children, researchers (Domitrovich, Cortes, \& Greenberg, 2007) designed a social-emotional training program geared to the developmental needs of preschoolers. Compared to children who did not have this training, those children who experienced the training were found to have a higher level of emotional knowledge, were rated by both teachers and parents to have gained more in terms of social competence, and were rated by teachers as exhibiting fewer signs of withdrawal in social situations (Domitrovich, Cortes \& Greenberg, 2007).

Ulatas and Omeroglu (2007) developed an education program for six year old preschoolers in Turkey. The educational training program was specifically designed to help the children to increase their skills at acknowledging, identifying, and finding appropriate responses to their emotions. The researchers found that the training did increase the measured level of emotional intelligence of the children who received the training when compared to either the control group or the placebo group (Ulatas and Omeroglu, 2007).

With respect to the middle school level, Wall (2005) reported on a clinical and educational project in a Boston school in which Tai Chi and elements of mindfulness-based stress reduction were taught to a small group of boys and girls. After the training, subjective statements by the participating children suggested that they felt greater calm, had an increased sense of selfawareness, and felt a stronger sense of connection with nature.

An intervention program was developed by 
Qualter, Whitely, Hutchison, \& Pope (2007) to enhance the level of emotional intelligence by primary school children preparing to transition to secondary school. This training was found to be particularly successful in aiding the transition for students who initially demonstrated lower levels of emotional intelligence.

Research by Yilmaz (2009) suggests that educational efforts can help develop abilities associated with emotional intelligence. In the study conducted by Yilmaz, Turkish college students were given training specifically to address the various dimensions of emotional intelligence identified by Goleman (1995) as described above. Pre and post research assessment of anger levels indicated a significant reduction in the anger levels of these students as compared to other students who did not experience the training.

Kremenitzer (2005) developed a system for selfreflective journaling for childhood teachers to track and record their own emotional reactions and awareness with regard to their teaching activities. Journaling offered teachers a specific path by with to build on the four areas of emotional intelligence identified by (Mayer, Salovey \& Caruso, 2004). In order to journal about their emotional reactions, the teachers needed to be aware of their emotions, consider the meaning and implications of those emotions in relation to the children they were teaching, and reflect on just how and whether they wanted to respond in the classroom in a way that would be most effective and beneficial for the students' learning process.

\section{Emotional intelligence in Nepali context}

Nepalese has been practicing on the basis of religion, culture and traditions as key components to learn emotional intelligence. The Hindu religions worship different gods and goddesses according to their character. The characters show their emotions like aggression (krodha) as of Shiva, relaxed and calm (baikuntha) as of Bishnu, and angry (krodha) goddess as Kali. The sculptures and paintings of these gods and goddesses also represent their emotional status. People worship them due to their positive qualities and their divine power for the truth.

The ancient religious stories also give knowledge about different types of emotions. Ramayan explained about love, joy, sadness, courage, anger, disgust, etc. This is best known for love between sibling's i.e Ram, Laxman and Bharat. When Raja Dasharath sent Ram to banabas (jungle) for 14 yrs, Laxman joined Ram and Sita because of extreme love between the siblings. Similarly, Bharat placed wooden shoes (kharau) on Singhasan (state chair) for all those years and worshiped the shoes with love and respect for the 14 years as acting head of state on Ram's behalf. It also shows the courage very nicely. After Rawan kidnapped Sita the courage showed by Ram and Laxman brought Sita back. It's because of their courage they were able to get help from other creatures like monkeys. Each character of Ramayan represents different emotions. Ram represents calm, Laxman represents aggression and love, Sita represents love, disgust (toward Rawan), Bharat represents love, surprise (with the decision taken by his father to send Ram to Banabas) etc.

Similarly, Mahabharat oriented people on emotional intelligence. It mostly exhibits jealousy, disgust and anger through different characters and episodes. Reading and watching such stories help children to understand how people can feel and react in different emotions.

Regarding the culture, the Nepalese observe different festivals and celebrations that also offer an opportunity to learn more on emotional intelligence. Dashain, Tihar, Jatra, Mother's day, Father's day, Teej, marriage ceremony and death ceremony are key. As previously described, emotional intelligence includes the ability to be self aware of one's emotional state, to make meaning of emotional reactions, and to regulate and manage emotional reactions (Mayer \& Salovey, 1990). Similarly Nepalese 
children might be self aware of god and goddess emotional state through religious stories and through sculptures. They could make meaning of emotional reactions and might manage emotional reactions. Due to different religious values norms and cultural aspects Nepal has diverse resources to develop emotional intelligence in a child. The child rearing practice itself can be a highly remarkable expression of emotional intelligence. While rearing a child the family pattern, norms and values, culture etc determines in emotional intelligence and it teaches a child about emotional intelligence.

While visiting in death ceremony people often express their sadness by crying. Therefore, people are encouraged to express their repressed and suppressed feelings openly as a psychological treatment. With references to all Nepalese traditional, cultural, and rearing practices, Nepalese children might develop good emotional intelligence however the individual differences do matter in developing emotional intelligence. Because of individual differences there are varied levels of emotional intelligence within a family, within siblings and parents. The practical aspects of working with emotional intelligence in one Nepalese counseling center will be described.

\section{Ankur Counseling Center and emotional intelligence}

Friends of Needy Children (FNC) established Ankur Counseling Center (ACC) as a sensitive entity. It has been working in psychological counseling since 2006 with an aim of improving the quality of life of FNC children through psychosocial care, training and education. Symptoms including depression, anxiety, withdrawal and extreme forms of acting out behaviors are the major dealing of ACC. It has been providing counseling services to the children and youth supported by Friends of Needy children (FNC), the Nepalese youth Opportunity Foundation (NYOF) and Happy House Foundation (HHF), as well as people of community.
In the beginning ACC counselors review referral forms and then schedule the clients for psychological counseling sessions. Thorough assessment information is gathered in different aspects such as past history; family background; physical and social issues; physical, sexual, or emotional abuses; and psychological tests relevant to the circumstances. During the psychological testing, ACC counselors often give I.Q. and E.Q. tests as per nature of problem.

Working ethically and effectively with emotional intelligence requires specialized training and supervised practice. ACC counselors have received advanced training as well as supervised practice in play and sand play therapy. Such training may begin with theory, but also requires guided supervision for practical application.

While observing emotional intelligence in a child, counselors allow children to perform some specified task in interaction with the counselor. Play therapy is one of the best tools to observe emotional intelligence of a child. Especially with children ACC counselor use play therapy such as marbles playing, storytelling, story completion, story making, role-play, emotion wheel etc. On story completion there are different types of intervention skills. Counselors provide children with a beginning and end of the story and the child has to finish the story. When the story is completed, counselor and child discuss how they feel and what might happen. In this way the counselor gathers information about the child's emotional intelligence. As described above, research suggests that children with average to high levels of emotional intelligences obtain higher grades and cause teachers fewer concerns about their behaviors (Qualter, et al., 2007). This appears to be true in the context of Nepal. Children are often discriminated by their performance intelligence. Some children were referred to ACC by on the basis that they are poor in academic achievement without reference to emotional intelligence. Therefore, to understand the emotional intelligence of 
children ACC has been using storytelling, story making, and story completion as key tools of emotion exploration. Group counseling sessions and specific observations in social events and celebrations actually help to identify E.Q.

In addition, ACC counselors use sand play therapy through which they can observe and understand the emotions of children. Sand play is an effective play therapy. In this therapy children should pick different kinds of miniatures from miniature shelves. Children starts to select miniatures as they have been feeling at present then they create their world on sand tray. Regarding their world they could portray their past experiences either negatively or positively. Typically children portray original experiences on sand tray, then their preferred stories, and situations. This encourages children to reflect on their emotional lives and sets the stage for more making purposeful choices in managing those emotions, an approach consistent with the research by Zeidner, Mathews, Roberts and MacCann (2003). By understanding this fact, ACC counselors observe children during sand play therapy with respect to their expressions as well as facilitate them to understand and express emotions appropriately. ACC believe the play therapies are an effective intervention to penetrate children's suppressed and repressed emotions. ACC also focuses on solutions of their problem through different therapeutic interventions.

During play therapy and counseling sessions counselors focuses on emotional intelligence through their activities, responses and their feeling about particular issues. One counseling session is usually not enough to assess someone's emotional intelligence. So ACC counselors also visit houses and schools. They join in picnics, hiking and other extracurricular activities. This allows counselors to get a better sense of how children's emotional lives and means of emotional regulation have been shaped through their family experiences as suggested by Zeidner, Mathews, Roberts, and MacCann (2003). Children often learn about emotional intelligence and are able to express their emotions through socialization process thus ACC counselors visit and attend social events. Before visiting these events and at celebrations counselors need to take permission with responsible persons.

While visiting families and attending celebrations, ACC counselors give much attention to children's behavior, including the emotional and social aspects. Through various responses in different situations and with different persons as teacher, uncle, aunty, guardian, friends, siblings, these all might show child's emotional intelligence silently. The experiences and conclusions from the observation through celebrations and other events, ACC counselors start to focus on emotional assessment in depth. The findings could be strengths to reduce problems that children are facing and it might be valuable information for the further counseling sessions.

For example, ACC visited some children's houses on the auspicious moment of Teej (Fasting day celebration of women). On that day children were in happy mood, smile on their face, excited to see each other especially girls by wearing chura, pote, tika. Boys are mostly busy teasing girls. Girls and women easily express their suppressed and repressed feelings through singing songs and dancing.

\section{Conclusion}

ACC counselors recognize that I.Q. is not enough for children to succeed in their lives while emotional intelligence really matters to gain success. If Ram is gifted in intelligence (math, science, other geometric and scientific subject) but he is poor in emotional intelligence and avoids social gatherings, he is not able to express his feelings or understand the feelings of others so he might not able to achieve according to his true capabilities.

In general educators and teachers often define children's or learner's ability in a single way i.e. I.Q. Children have been punished due to their 
$42 \mid$ Christopher Rybak, Chbori Laxmi Maharjan \& Anubha Adbikari

low I.Q and expelled from school. Educators are not often able to give their attention to children's emotional intelligence as they express through art, drawings, singing, dancing, acting as well as various social behaviors. As teachers, educators and guardians need to be aware of modeling and helping children develop emotional intelligence. Therefore to understand children or someone's ability through eyes of intelligence we need to keep I.Q. and E.Q. in both our eyes. If one eye does not have sufficient vision then other eye could work effectively.

With respect to counselors the level of emotional intelligence affects their therapeutic competence in working with children as well as adults (Easton, Martin, \& Wilson, 2008). Therefore to understand an individual's emotional intelligence a counselor should have well-developed emotional intelligence in different aspects. As described by Miville, et al (2006), effective counselors empathically identify the emotions experienced by clients while maintaining clarity about their own emotional state. ACC counselors believe that children do have their own special ability and they should not be labeled according to one type of intelligence because there are other aspects where we need to be sensitive. These problems and challenges can reduce through psychological counseling and therapies. A key to effective counseling in this and other applications requires a commitment to continual learning as well as supervised practice with competent and well-trained practitioners.

\section{References}

Domitrovich, C. E., Cortes, R. C., \& Greenberg, M. T. (2007). Improving young children's social and emotional competence: A randomized trial of the "PATHS" program. The Journal of Primary Prevention, 28, 67-91. doi: 10.1007/s10935-007-081-0.

Easton, C., Martin, W. E. \& Wilson, S. (2008). Emotional intelligence and implications for counseling self-efficacy: Phase II. Counselor Education \& Supervision, 47, 222-232.

Goleman, D. (1995). Emotional Intelligence: Why it can matter more than IQ. New York: Bantam.

Killgore, W. D. S., \& Yurgelun-Todd, D. A. (2007). Neural correlates of emotional intelligence in adolescent children. Cognitive, Affective, and Behavioral, Neuroscience, 7, 140-151.

Kremenizter, J. P. (2005). The emotionally intelligent early childhood educator: Self-reflective journaling. Early Childhood Education Journal, 33, 3-9. Doi: 10.1007/s10643-005-043-6.

Martin, W. E, Easton, C., Wilson, S., Takemoto, M., \& Sullivan, S. (2004). Salience of emotional intelligence as a core characteristic of being a counselor. Counselor Education \& Supervision, 44, 17-30.

Mavroveli, S., Petrides, K. V., Sangoureau, Y., \& Furnham, A. (2009). Exploring the relationships between trait emotional intelligence and objective socialemotional outcomes of childhood. British Journal of Educational Psychology, 79, 259-272.

Mayer, J. D., \& Salovey, P. (1990). Emotional intelligence. Imagination, Cognition, \& Personality, 9, 185-211.

Mayer, J. D., \& Salovey, P. (1995). Emotional intelligence and the construction and regulation of feelings. Applied \& Preventive Psychology, 4, 197-208.

Mayer, J. D., Salovey, P., \& Caruso, D.R. (2004). Emotional intelligence: Theory, findings, and implications. Psychological Inquiry, 15, 197-215.

McElwain, N. L., Halberstadt, A. G., \& Volling, 
B. L. (2007). Mother- and fatherreported reactions to children's negative emotions: Relations to young children's emotional understanding and friendship quality. Child Development, 78, 14071425 .

Miville, M. L., Carlozzi, A.E., Gushue, G. V., Schara, S. L., \& Ueda, M. (2006). Mental health counselor qualities for a diverse clientele: Linking empathy, universal-diverse orientation, and emotional intelligence. Journal of Mental Health Counseling, 28, 151-165.

Posner, M. I., \& Rothbart, M.K. (2007). Education, psychology, and the brain. In M. I. Posner \& M. K. Rothbart (Eds.) Educating the human brain ( pp. 7-24). Washington, D.C.: American Psychological Association.

Qualter, P., Whitely, H. E., Hutchison, J. M., \& Pope, D. J. (2007). Supporting the development of emotional intelligence competencies to ease the transition from primary school to high school. Educational Psychology in Practice, 23, 79-95.

Rizzolatti, G. \& Fabbri-Destro, M. (2008). The mirror system and its role in social cognition. Current Opinion in Neurobiology, 18, 179-184.

Romasz, T. E., Kantor, J. H., \& Elias, M. J. (2004). Implementation and evaluation of urban-wide social emotional-learning programs. Evaluation \& Program Planning, 27, 89-103.

Rybak, C., \& Earhart, R. (2008). A path toward healing: Children coping within a hostile environment. In H.L. Kaila (ed.) Anger in children: Causes, characteristics and considerations (pp. 21-37). New Delhi: MD Publications Pvt. Ltd.

Siegel, D. J. (2007). The mindful brain: Reflection and attunement in the cultivation of wellbeing. New York: Norton \& Company.

Ulatas I., \& Omeroglu, E., (2007). The effects of an emotional intelligence training program on the emotional intelligence of children. Social Behavior \& Personality, $35,1365-1372$.

Wall, R. B. (2005). Tai chi and mindfulnessbased stress reduction in a Boston public middle school. Journal of Pediatric Health Care, 19, 230-237.

Yilmaz, M. (2009). The effects of an emotional skills training program on the consistent anger levels of Turkish university students. Social Behavior \& Personality, 37, 565-576.

Zeidner, M., Mathews, G., Roberts, R. D., \& MacCann, C. (2003). Development of emotional intelligence: Towards a multi-level investment model. Human Development, 46, 69-96. 